\title{
Real-Time Wildfire Monitoring and Alert System Using GSM Technology
}

\author{
Aniedu A. $\mathrm{N}^{1}$, Chukwuneke C.I ${ }^{2}$, Asogwa D. $\mathrm{C}^{3}$, Nwokoye C. S. ${ }^{4}$ \\ ${ }^{1,4}$ Department of Electronics and Computer Engineering, NnamdiAzikiwe University, Awka, \\ ${ }^{2,3}$ Department of Computer Science, NnamdiAzikiwe University, Awka
}

\begin{abstract}
Environmental related issues and parameters are of utmost importance to man, and is essential to his existence and influence and thus he alwaysseeks for an improved system that would be able to capture and monitor the changes in environmental parameters irrespective of time and location so as to provide for measures that will forestall abnormalities and cater for emergencies.This paper presents research work on a system that is capable of providing real time remote wildfire monitoring and SMS alert. Thework aimed at the design and implementation of a low cost but efficient and flexible wildfire monitoring and alert system using GSM technology. It was designed in such a way that the Monitoring of wildfire would be achieved with the use of temperature and smoke sensorscoupled with a control unit and transmitter module all of which are battery powered. Fire and smoke are sensed and measured by the sensors which send the signals to the control unit for proper processing and determination of the smoke rate.This is displayed on an LCD screen, then an alert is sent to the mobile phone of the environmental personnel via SMS while simultaneously triggering an alarm in control room. Thus this system presents a continuous, real time, remote, safe and accurate monitoring of wildfires and smoke rate hence ensuring the conservation and preservation of wildlife, natural habitats and the ecosystem, and at the same time, helping tocurb high impact human, industrial and environmental damage
\end{abstract}

Keywords: Wildfire, Temperature sensor, Smoke rate, GSM Technology, SMS, Real time monitoring, Emergencies.

\section{Introduction}

Environmental, Industrial and home hazards has been identified as the main causes of deaths, disabilities, deformation and natural loss in many countries and thus it accounts for millions of deaths and losses worldwide. The delay between the first signs of smokes and fire outbreak with respect to emergency callsfor response and intervention has a large variation among different situations and locations resulting to fatal consequences. Hence with evolution and advancement of communication technologies, new strategies are being adopted in order to reduce delays and ascertain rate of impact for appropriate response. Wildfire Monitoring is one possible solution. Wildfires are expensive disasters in terms of both property loss and life safety. Wildfire often occurs in environmental sensitive regions such as forest, park, and grassland area or along the urban-wild land interface. Environmental monitoring in such terrain must be environmentally appropriate, which requires easy installation, low maintenance and relatively inexpensive instrumentation [1].Forest fires, also known as wildfires are uncontrolled fires occurring in wild areas and can cause significant damage to natural and human resources. Forest fires eradicate forests, burn the infrastructures, and may result in high human death toll near urban areas. Common causes of forest fires (wildfires) include lightning, human carelessness, and exposure of fuel to extreme heat and aridity. It is known that in some cases fires are part of the forest ecosystem and they are important to the life cycle of indigenous habitats. However, in most cases, the damage caused by fires to public safety and natural resources is intolerable and early detection and suppression of fires deems crucial [2].Residential places are also prone to wildfire disasters which may lead to loss of lives and properties. Industries, offices, and companies are also susceptible to fire outbreak due to some electrical faults in any of the machines or devices. For these unfavorable incidents to be prevented there is a need for wildfire monitoring systems. Fire sensors and smoke sensors can be placed in some places where there are possibilities of having fire outbreak for better monitoring of the industry or offices or company against fire outbreaks in order to prevent further fire disaster within the area of occurrence.

One of the most effective reliable and trendy techniques of monitoring wildfires occurring in remote places is theGSM technology.The global system for mobile communication (GSM) is a digital wireless technology standard based on the notion that users want to communicate wirelessly without limitations created by geographic or national borders. In a short period of time, GSM has become a global phenomenon. The temperature and smoke sensors are used for gathering environmental information which is transmitted to the receiver module of the GSM module in order to call for the attention of the fire-fighters.In this device, the smoke and fire are sensed and detected by using sensors as analog data, then it is converted into digital data using analog to digital converter (ADC) which is suitable for wireless transmission using SMS messages 
through GSM modem. The control unit is used for temporary storage of the data used for transmission. This work focused on the design of the fire monitor that is able to monitor the smoke rate continuously and can be used in hospitals, homes, industries, parks, public place, nature parks and forest.

\section{Background Study}

Recent breakthroughs in science and technological innovations have led to an unprecedented advancement in provisions of technological solutions for the numerous problems facing mankind. Researchers are busy leveraging on modern technology to provide better and improved solutions commensurate to the ever increasing demands. In an environment there are parameters to be monitored such as temperature and smoke etc. which are evidences or signs of presence of fire within an environment. Temperature and smoke of an environment can rise as a result of heat which can stem from vast number of both identifiable and unknown sources. If all these sources of heat are not properly monitored and controlled there will be uncontrolled rise in temperature which will result to fire outbreaks. Therefore anything that generates heat also produces smoke as it gets contact with some materials. The presence of smoke shows that the material has been heated up immensely and this causes change in the atmosphere of the environment thereby causing air pollution. A fire or smoke detector is a device that allows ones to sense and detect the presence of smokes and fires and allows one to record or store the rate for later study, further analysis or authenticates an onward transmission for immediate response. Sensor fusion algorithms allow these monitors to detect core temperature, heat and dehydration. The digital smokes and fire detectors and alert systems provides a more unique, effective and efficient means of realtime monitoring of smokes and fire and has ever since witnessed an unprecedented tremendous advancement as researchers keep searching for better ways to make these fire monitoring and alert systems more flexible, portable, and efficient. This section presents a review of current research findings and works done so far by different researchers with the same mindset of providing flexible, portable, and efficient monitoring and alert systems.

\section{Review of Related Works}

In a work done by [3] real-time remote wildfire monitoring system was developed for forest using ZigBee based wireless sensor network.Zig-Bee is a Technological Standard Created for Control and Sensor Networks based on the IEEE 802.15.4 specification for wireless personal area network .It is a newwireless technology that has application in various fields. This technology utilizes zig-bee modules in remote monitoring applications which operate in the ISM (industrial, scientific and medical) Band utilizing the zig-bee protocol created by zig-bee Alliance. They are suitable for Wireless Personal Area Networks (WPAN) but can extend a broader coverage area using multi-hop routing technology.The main features of this standard arenetwork flexibility, low cost, very low power consumption, and low data rate in an ad-hoc self-organizing networkamong inexpensive fixed, portable and moving devices.

In a work done by [4], real-time remote wildfire monitoring and control system was developed using GSM technology and fire extinguisher vehicle. The temperature sensor sends an analogue signal to the PIC microcontroller to set off the alarm when the temperaturereaches $125^{\circ} \mathrm{F}$. The design consists of a temperature sensor (LM-35) whose output voltage is equal to $1^{\circ} \mathrm{F} / 10 \mathrm{mV}$. The smoke sensor is a $\mathrm{T}$-shaped chamber with an infrared LED placed in the left opening that emits a beam of lightacross the horizontal portion of the chamber. When smoke particleenter the T-shaped chamber, however the beam of light isscattered by smoke particles. Subsequently, some of the lightis directed down into the vertical portion of the T-shapedchamber and strikes the phototransistor.The smoke detection system can detect smoke or high temperature (potential fire) and sets off appropriate alarm sound. It also transmit RF signal to the other smoke detector in the network. The detector is capable of soundingfour different alarms for smoke, temperature, low battery andreceiver.

In another work done by [5], wildfire monitoring system was designed using wireless sensor network (WSN) technology (fig 1). This system should be able to detect a small fire (about tens of meters in size) and determine its position with accuracy sufficient to make possible a fast intervention and fire spread limitation. The detection cells will be connected via a wireless data transmission system with a central control or data collection point connected to the warning system of the emergency service. In this way, the immediate response fire alarm is realized.The fire monitoring WSN is based on Mica2 motes and MTS400 sensor boards. It uses the following components:Temperature, humidity, barometric pressure and light intensity sampling nodes, Routing nodes for transporting relative readings from the sampling nodes to the base station, Base-station connected to web server,Web server connected to a MySQL database, which is queried by a browser-based client,Client providing data browsing, GPS information and fire alarm, sending to fire-fighters to response to the fire. 


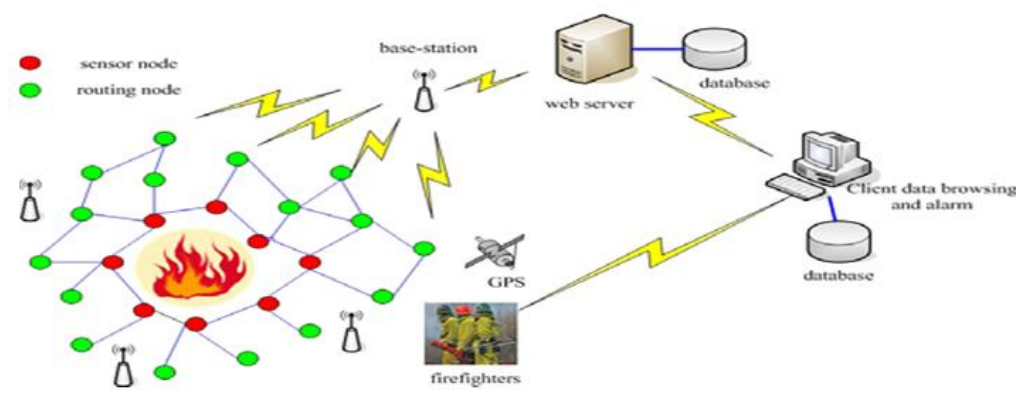

Fig 1: The Architecture of WSN for Wildfire Monitoring

In usual time, environmental data are collected by a few sensor nodes in a low frequency when it is humid with low fire possibility. When a fire occurs, the nodes surrounded are activated to sample related data such as temperature, humidity and barometric pressure in a relatively high frequency. The collected data are relayed through routing nodes and base-station to be stored into a MySQL database connected to web server, which is queried by a browser-based client. Data information are processed or aggregated in the base-station to reduce the communication cost. Client provides effective information including fire extent and location for firefighters to make responsive action.

In a work done by [6], wireless fire alarm system was constructed using Bluetooth wireless technology. Bluetooth is wireless standard technology invented by Telecom vendor Ericsson in 1994 for exchanging data over short distances from fixed and mobile devices and building personal area network. It was originally conceived as a wireless alternative to RS-232data cables. It can connect several devices, overcoming problems of synchronization. Bluetooth is managed by the Bluetooth Special Interest Group (SIG), which has more than 19,000 member companies in the areas of telecommunication, computing, networking, and consumer electronics. Bluetooth operates in the range of $2400-2483.5 \mathrm{MHz}$. Bluetooth exists in many products, such as telephones, tablets, handheld and console gaming equipment, and some high definition headsets, modems, and watches. By using fire alarm project one can use Bluetooth device for transferring data from fire alarm system to computer.In this one will be capable of performing the function of fire alarm and make use of computer as control panel to monitor the temperature. With Bluetooth technology it can penetrate obstacles. With the help of control panel the user can check the surroundings temperatureat any time and make analysis on their own from database that is recorded. If the Bluetooth fails in this case we can use buzzer when temperature is over limits. The advantages of using Bluetooth technology are as follows: Enhances user's experience, Connecting devices without use of cables, Reduced power consumption, Industry wireless communication standard, It is affordable.

In work done by [6], Wi-Fi based wireless fire alarm system was developed. Wi-fi is also a wireless technology that allows electronic device to exchange data or to connect to the internet wirelessly using $2.4 \mathrm{GHz}$ UHF and 5 SHF radio waves. A Wi-Fi-enabled device can connect to the Internet when within range of a wireless network which is configured to permit this. The coverage of one or more access points called hotspots can extend from an area as small as a few rooms to as large as many square kilometers. Wi-Fi allows cheaper deployment of local area networks.Wi-Fi alarms are the ideal solution for applications in small to medium sized commercial enterprises, offices, car showrooms, schools, village halls and meeting rooms, sports and leisure facilities, and many other applications. Typical Wi-Fi alarm systems incorporate detection devices, manual call points, alarms or sounders, and control panels with remote keypads. Alarms can also be activated and deactivated with a single press of a button on individually programmed key fobs at up to $50 \mathrm{~m}$ from the control panel. Reducing damage and complex installation make Wi-Fi alarms an attractive proposition amongst a growing number of users. The latest Wi-Fi systems incorporate attractively designed individual components that complement the interiors of both modern or traditional premises and properties. Typical Wi-Fi specifications are user-friendly LCD display, radio controlled panel with unique connections for up to $100 \mathrm{Wi}-\mathrm{Fi}$ devices, bidirectional radio control and alarm functions, individual zones with separate identification of the device activated, control / monitoring and testing functions for all radio devices, integrated power supply for $230 \mathrm{~V}$ connection, and internal battery back-up.

From the literatures reviewed, it can be seen that real-time remote fire (wildfire) monitoring has been implemented for environments or buildings using various technologies such as GSM/GPRS technology, Wireless sensor network, Bluetooth wireless technology,andZig-bee based wireless sensor network technology. Each of this technology has its own set of advantages and disadvantages and the deployment of any of these technologies in an environment is dependent on the availability of the components needed to build the system, the coverage zone and the intended mobility of the operator. Bluetooth wireless technology can exchange data over short distances such as 10 meters from fixed and mobile devices and it cannot be used for large area of coverage. Zig-bee, Wi-fi, WSN technology, GSM/GPRS, and RF module can be used for long distances applications especially in real-time remote wildfire monitoring system.

DOI: 10.9790/0050-03040110 www.iosrjournals.org $3 \mid$ Page




\section{A. Proposed System}

This works presents a lot of considerations and improvements that were incorporated in to the functionality of the device so as to reflect desired features such as cost, design complexity, size, software development, weight, portability etc. This design uses a miniaturized smoke sensor (IC sensor) which has been optimized for very accurate sensing and smoke detection. The system determines the rate and intensity of fire and smoke and with the help of the control unit displays the detected rate on a 16X2 character LCD and sends an SMS, each time the smoke rate goes above or below a fixed threshold, while at the same time setting off a buzzer alarm attached to the device to trigger an alert. With small size and portability in mind, the choice of the LCD display and miniaturized sensor aims at eliminating the need for a PC display, It thus ensures flexibility in real-time remote monitoring regardless of distance and location.Another interesting feature of this particular design is the reprogrammable and open source nature of the product, which makes it easier to re-specify the particular heat rate to watch out for, as well as play with the system parameters, to suit the users' needs better. This is necessary due to varying environmental and patient conditions. The introduction of the open source Arduino board in this project makes it exceptionally unique and thus opens door for greater exploration and maximization of its great flexibility features and the extent to which it can be implemented for a variety of functions. The overall block diagram of thesystem is shown in Figure 2:

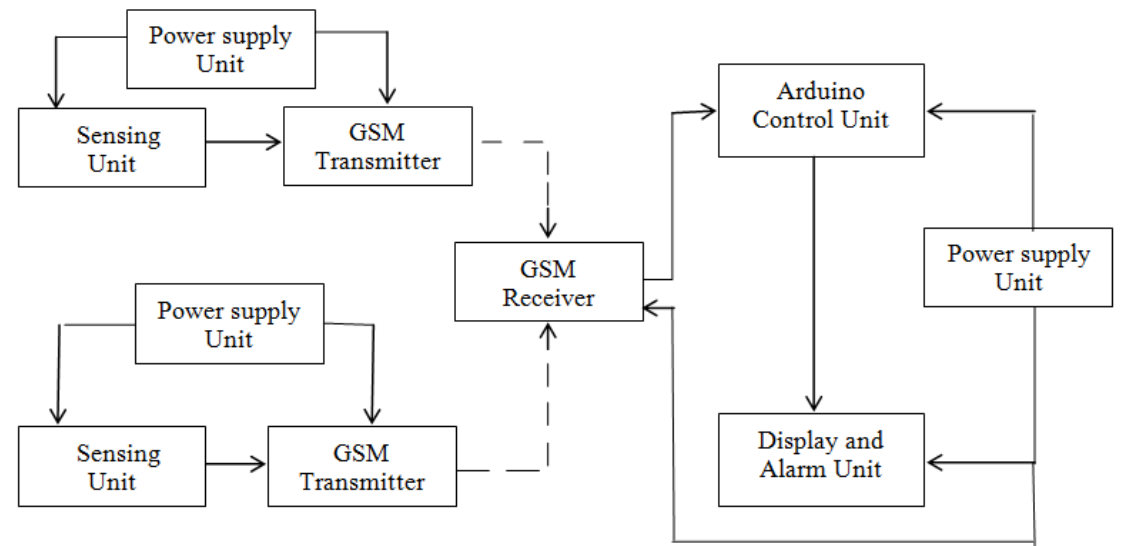

Fig 2:Block diagram of the overall system

\section{Hardware Subsystem Description}

\section{System Design}

\section{A. Sensing Unit}

Two types of sensor nodes are utilized in this project namely:

Coordinator node: This node will act to receive temperature or smoke readings from the various end sensor nodes and convey signals to the LCD and buzzer through AT89C52 microcontroller.

The various hardware Components/Units required for the implementation of the Coordinator node are:

Power Supply Unit, RF Receiver module Unit, Decoder unit, Microcontroller/Processing Unit, LCD display and Alarm Units. The arrangement of the Coordinator node is depicted in figure 3:

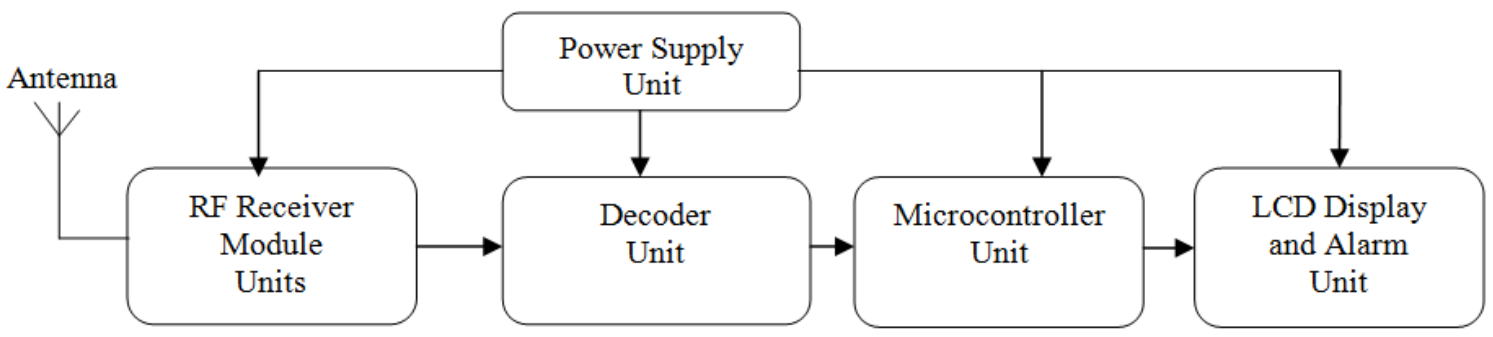

Figure 3: Block diagram of the Coordinator node

End Sensor node: This node will act to acquire temperature and smoke readings from the monitoring environment and transmit them to the coordinator node.The various hardware Components/Units required for the implementation of the End Sensor node are:Power Supply Unit, Sensing unit, Encoder Unit, RF Transmitter module Unit,The arrangement of the end sensor node 1 is shown in Figure 4. End Sensor node 2 is exactly similar to end sensor node 1 . 


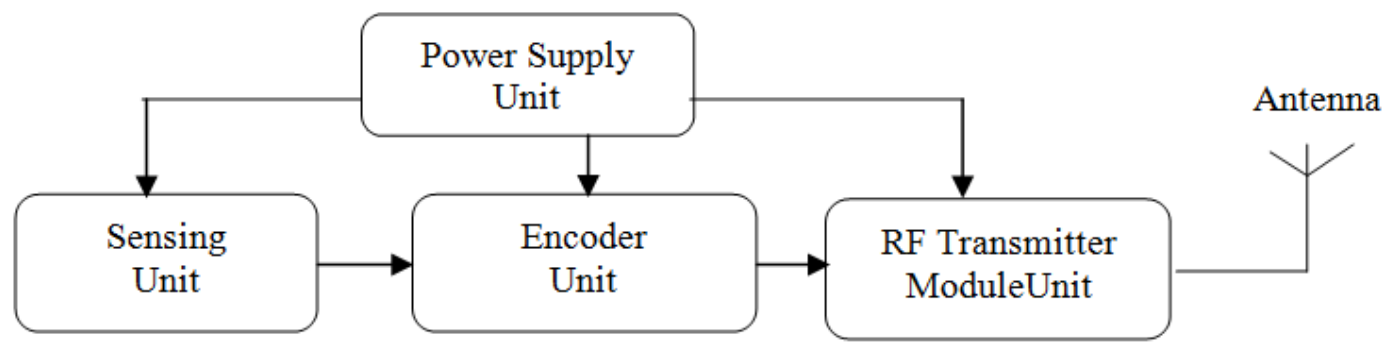

Figure 4: Block Diagram of the End Sensor Node 1

This unit is concerned with acquiring of the temperature readings from the environment where it is installed. For this work, this system makes use of a temperature sensor LM 35. The LM 35 Integrated Circuit (IC) is a precision temperature sensor with an output voltage linearly proportional to the centigrade temperature. Thus, the LM 35 has an advantage over linear temperature sensors in ${ }^{\circ}$ Kelvin as one does not require subtracting a large constant voltage from the output to obtain convenient centigrade scaling. Another advantage of the LM 35 is that it does not require an external calibration or trimming to provide accuracies of $\pm 1 / 4^{\circ} \mathrm{C}$ at room temperature. The LM 35 requires only about 65 microamperes from the supply and has a very low self-heating of $0.1^{\circ} \mathrm{C}$ in still air and operates over a full $-55^{\circ} \mathrm{C}$ to $+150^{\circ} \mathrm{C}$ temperature range [7].

Therefore, the formula to calculate the temperature reading from the LM 35 in Celsius is:

$\mathrm{T}\left({ }^{\circ} \mathrm{C}\right)=\ldots \ldots \ldots \ldots \ldots \ldots \ldots \ldots \ldots \ldots \ldots \ldots(1)$

The LM 35 sensor is an accurate temperature sensor already having inherent trimming. However, when the LM 35 sensor is used directly in the system, the readings will suffer from fluctuations in the power supply. To get more accurate readings, external trimming is required as shown in figure 4

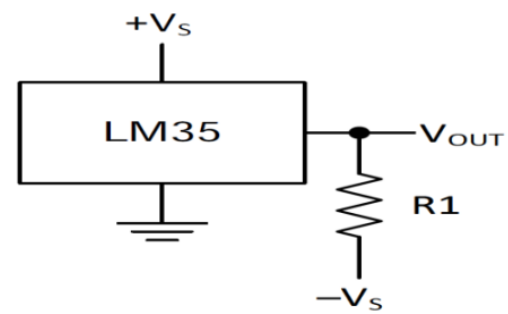

Fig 4: External resistor used in providing more accuracy to the LM 35 readings.

As shown in figure 3, a pull down resistor is used to provide more accuracy by pulling down any stray current from the circuit from affecting the output reading of the LM 35. The value of the pull down resistor R1 from LM 35 datasheet is calculated as:

$\mathrm{R} 1=\mathrm{Vs} /\left(50 \times \llbracket 10 \rrbracket^{\wedge}(-5)\right.$ amps $)$

Where $\mathrm{Vs}$ is the voltage source to the LM 35

$0.5 \mathrm{~mA}\left(50 \times \llbracket 10 \rrbracket^{\wedge}(-5)\right.$ A $)$ is chosen as the maximum value in which a stray current from the circuit due to power fluctuations will not exceed under normal circumstances.

In this project, $\mathrm{Vs}$ to the $\mathrm{LM} 35$ is $5 \mathrm{v}$. Therefore, the value of R1in this project is:

$\mathrm{R} 1=5 \mathrm{v} /\left(50 \times\left[(10)^{\wedge}(-5)\right.\right.$ A) $=1 \times[10) \wedge 4 \Omega$ or $10 \mathrm{~K} \Omega$

The LM 35 produces a voltage output of 10 millivolts $(\mathrm{mV})$ for every $1{ }^{\circ} \mathrm{C}$ rise in temperature.

This unit also consists of smoke sensor module (MQ2) and it is used for gathering information about the gaseous changes in an environment which indicates that fire is suspected (fig 4). A smoke sensor is a device that senses smoke, typically as an indicator of fire. The Analog Smoke/LPG/CO Gas Sensor (MQ2) module utilizes an MQ-2 as the sensitive component and has a protection resistor and an adjustable resistor on board. The MQ-2 gas sensor is sensitive to LPG (Liquidified petroleum gas), i-butane, propane, methane, alcohol, Hydrogen and smoke. It could be used in gas leakage detecting equipment in family and industry. The resistance of the sensitive component changes as the concentration of the target gas changes [8]. Features of the MQ2 smoke sensor include: Dimension: $32 \mathrm{~mm}$ x $22 \mathrm{~mm}$ x 30mm Specification, Operating voltage: $5 \mathrm{v}$, Detection Zone: 300 - 10000ppmm, Characteristic Gas: 1000ppmm, Sensitivity: R in air/ R in typical gas > 5, Response Time: <10s, Recovery time: <30s, Heating Resistance: 31ohm, Heating Current: <181mA, Heating Power: $<900 \mathrm{~mW}$, Measuring Voltage: <24, Ambient Temperature: -20C - 55C, Humidity: <95\%, Oxygen Content: $21 \%$. 
The circuit diagram of the entire sensing unit is shown in figure 5

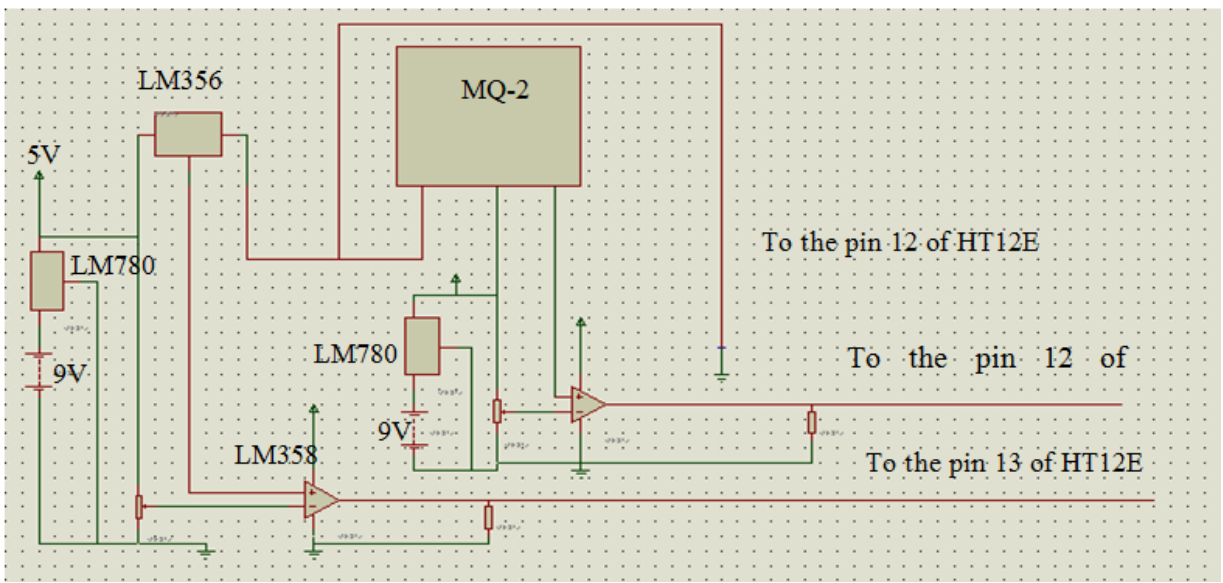

Figure 5: Circuit diagram of the entire sensing unit

\section{B. Control Unit (The Arduino Board)}

The Arduino Uno is a microcontroller board based on the ATmega328 (datasheet). It has 14 digital input/output pins (of which 6 can be used as PWM outputs), 6 analog inputs, a $16 \mathrm{MHz}$ ceramic resonator, a USB connection, a power jack, an ICSP header, and a reset button. It contains everything needed to support the microcontroller; simply connect it to a computer with a USB cable or power it with an AC-to-DC adapter or battery to get started. It's an open-source physical computing platform based on a simple microcontroller board, and a development environment for writing software for the board.

The Basic Features of Arduino Board

- The micro-controller used is ATmega328, built with Harvard architecture advanced RISC (Reduced Instruction Set Computing) technology.

- Operating voltage is $5 \mathrm{v}$.

- The recommended input voltage is $7-12 \mathrm{v}$.

- Input power supply voltage range: $6 \mathrm{v}-20 \mathrm{~V}$.

- Digital I/O Pins are 14 (of which 6 provide PWM output).

- The analog input pins are 6.

- The DC Current per I/O Pin is $40 \mathrm{~mA}$.

- The DC Current for 3.3V Pin is $50 \mathrm{~mA}$.

- The Flash Memory is $32 \mathrm{~KB}$ of which $0.5 \mathrm{~KB}$ used by boot loader.

- $\quad$ The SRAM size is $2 \mathrm{~KB}$.

- The EEPROM size is $1 \mathrm{~KB}$.

- $\quad$ The Clock Speed is $16 \mathrm{MHz}$

\section{C. $\quad$ RF (Radio Frequency) module Unit}

An RF Module is usually a small electronic circuit which is used to transmit and/or receive radio signals on a number of carrier frequency. RF modules are widely used in electronic design owing to the difficulty and complexity of designing radio circuitry. This good electronic radio design is very complex only because of the sensitivity of radio circuits and the accuracy of components and layouts that are required to achieve operation on a specific frequency.

This RF module comprise of a RF Transmitter and an RF Receiver. The transmitter/receiver (Tx/Rx) pair operates at a frequency of $434 \mathrm{MHz}$. This RF module used in this project employs Amplitude Shift Keying transmission scheme. A RF transmitter receives serial data and transmits it wirelessly through RF through its antenna is connected at pin4. Transmission takes place at the rate of $1 \mathrm{Kbps}-10 \mathrm{Kbps}$. The transmitted data is received by an RF receiver operating at the same frequency like the transmitter. This RF module allows one way communication between two nodes that are known as transmission and reception. The RF module is often used along with a pair of encoder and decoder. Encoder is used for encoding parallel data for transmission feed while reception is decoded by a decoder. HT12E and HT12D are commonly used encoder and decoder pair ICs respectively and they are used in this project. The RF Receiver module and RF Transmitter module pin diagram is shown below in figure 6 


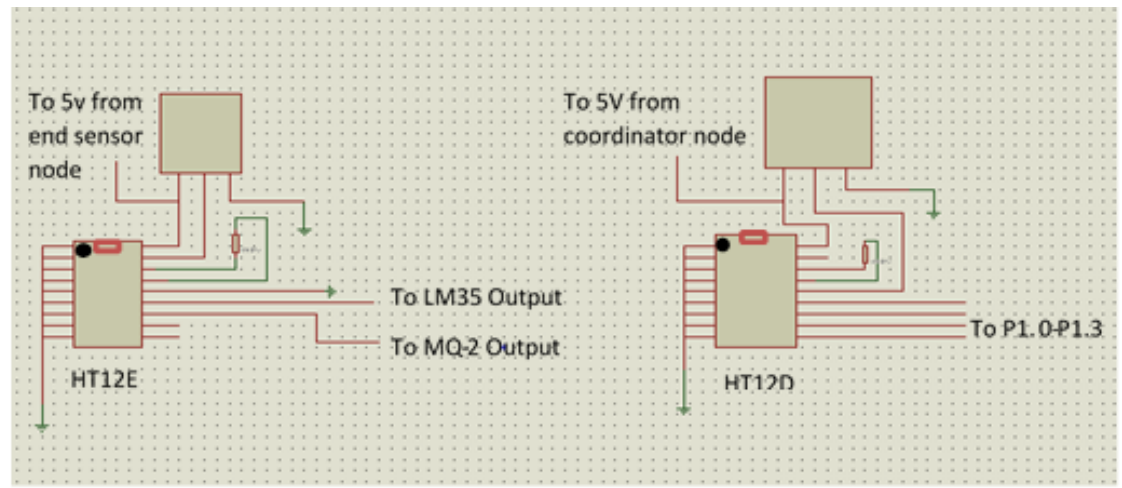

Figure 6: Circuit diagram of RF module Unit

\section{Processing Unit}

The processing unit of this project is the AT89C52 microcontroller, it processes the parallel data received from the HT12D decoder and controls the operation of both the Liquid crystal display and the piezoelectric buzzer. The display on the liquid crystal display when fire is detected depends on the programs written in assembly language which is burned into AT89C52 microcontroller chip. The sound made by the piezoelectric buzzer when fire is detected depends also on the programs written in C language which is burned into AT89C52 microcontroller chip.

The overall design of the circuit as generated from the software design tool is shown in figure 7.

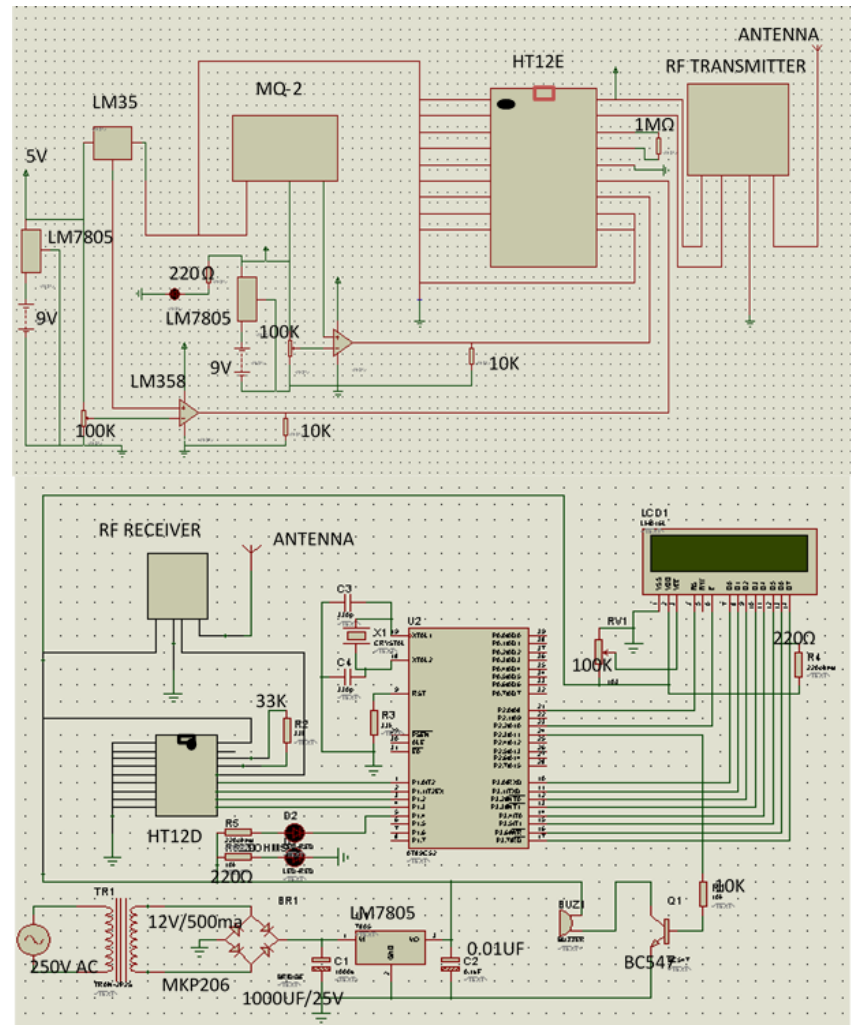

Fig. 7: Overall circuit diagram of the system

\section{Software Subsystem Design} below;

The control unit was initialized and programmed using the system flowchart procedure enumerated

Step 1: Monitoring if the temperature of the environment has increased.

Step 2: Monitoring if there is smoke in the environment.

Step 3: Transmission takes place when there is increase in temperature and smoke in the environment.

Step 4: Reception takes place immediately transmission is done.

Step 5: Microcontroller displays fire message and triggers an alarm 
Figure 8depicts the operation and flow chart of the whole system:

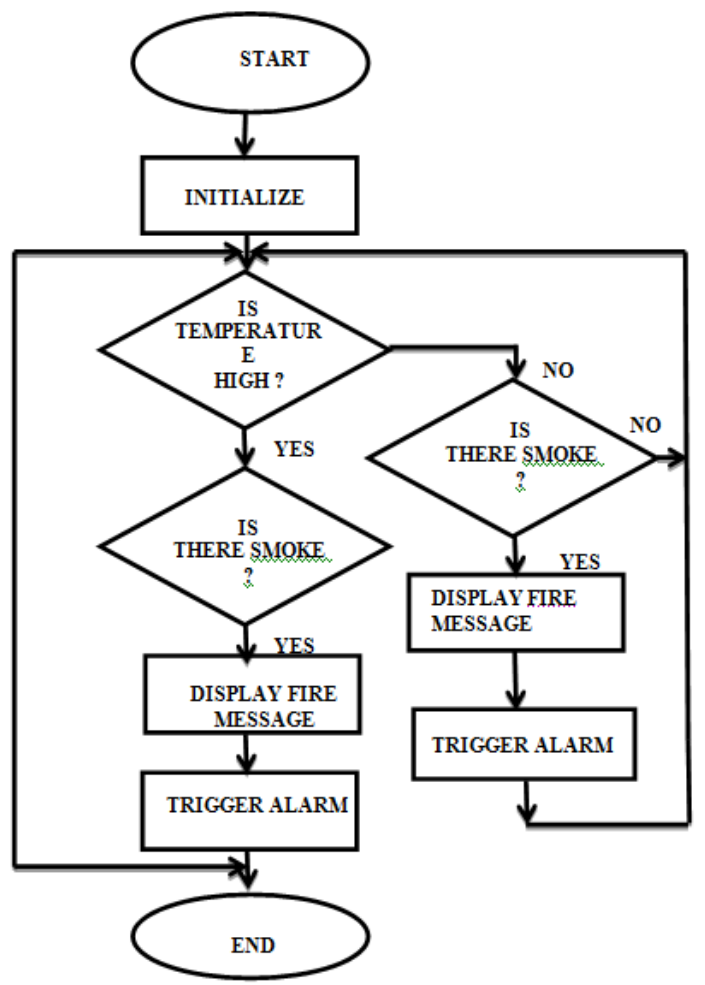

Fig 8: System Flowchart

V. System Implementation, Analysis and Evaluation

The individual unit of the entire system was properly tested before full implementation was carried out. Various testing conditions were applied to the components depending on their specifications. Fire and Electric cooker were used to test the output response of the LM35 sensors with respect to distance. The setup was implemented on bread board and the output was finally tested with multi-meter. The results obtained showed that as the temperature of the fire increases the output voltage of the LM35 sensor increases while that of the electric cooker increases as the output voltage of the LM35 sensor increases. The readings obtained are shown in Table1 while Figure 9 shows the graphical representation of the response.

Table 1: Readings of the test carried out on LM35 sensor

\begin{tabular}{|c|c|c|c|c|c|c|c|c|c|}
\hline Heat sources & $\mathrm{F}$ & & & 1 & & $\mathrm{r}$ & & $\mathrm{e}$ & E 1 e c t r i c \\
\hline Distance $(\mathrm{cm})$ & \multicolumn{8}{|c|}{ Voltage $(\mathrm{mV}) /$ Temperature $\left({ }^{\circ} \mathrm{C}\right)$} & Voltage $(\mathrm{mV}) /$ Temperature $\left({ }^{\circ} \mathrm{C}\right)$ \\
\hline 2 & 6 & 6 & 0 & / & $\begin{array}{ll}6 & 6 \\
\end{array}$ & ( & $\circ \mathrm{C}$ & & $\left.\begin{array}{lllllllllll}1 & 0 & 0 & 0 & / & 1 & 0 & 0 & \left({ }^{\circ}\right. & \mathrm{C}\end{array}\right)$ \\
\hline 4 & 3 & 5 & 0 & / & 35 & ( & $\circ \mathrm{C}$ & & $\left.\begin{array}{llllllllllll} & 6 & 7 & / & 9 & 6 & . & 7 & \left({ }^{\circ}\right. & \mathrm{C}\end{array}\right)$ \\
\hline 6 & 3 & 0 & 0 & l & $\begin{array}{ll}3 & 0 \\
\end{array}$ & ( & ${ }^{\circ} \mathrm{C}$ & 1 & $\left.\begin{array}{lllllllllll} & 5 & 2 & / & 7 & 5 & . & 2 & \left({ }^{\circ}\right. & \mathrm{C}\end{array}\right)$ \\
\hline 1 & 2 & 9 & 0 & l & 29 & ( & ${ }^{\circ} \mathrm{C}$ & & $6 \begin{array}{llllll}6 & 0 & 5 & / & 6 & 0\end{array}$ \\
\hline 2 & 2 & 8 & 0 & l & 28 & ( & ${ }^{\circ} \mathrm{C}$ & & $\left.\begin{array}{lllllllllll} & 6 & 5 & / & 5 & 6 & . & 5 & \left({ }^{\circ}\right. & \mathrm{C}\end{array}\right)$ \\
\hline
\end{tabular}
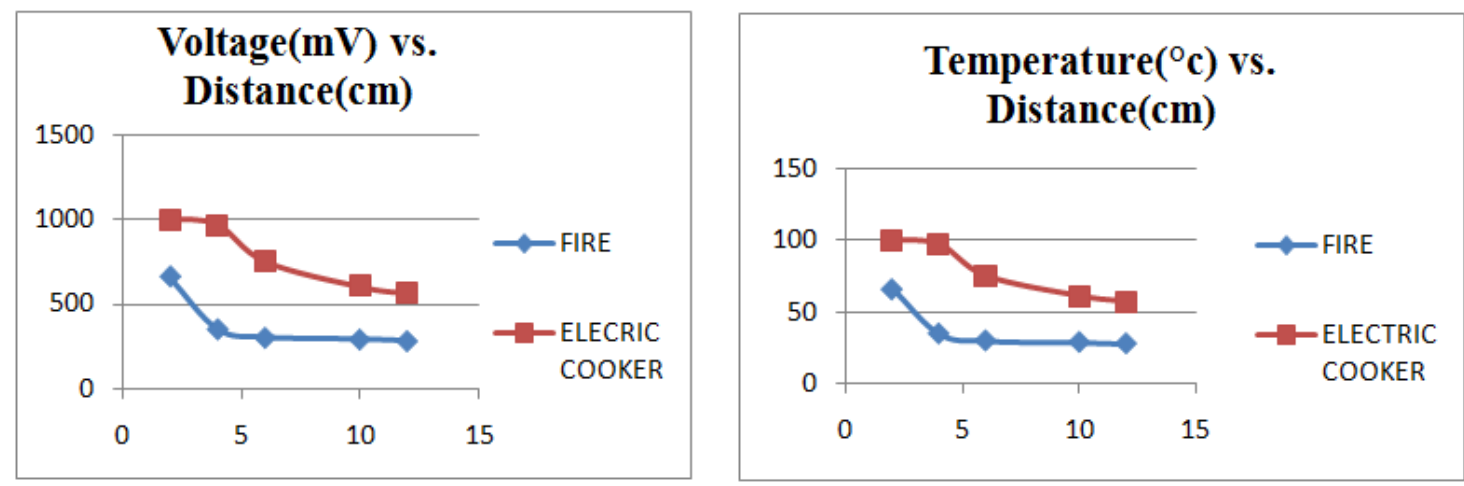

Fig 9: Graphical representation of the response of LM35 using fire and electric cooker 


\section{Testing of the MQ-2 Sensor}

MQ-2 (Smoke sensor) was tested by using multi-meter to measure the output voltage from the sensor when smoke from fire was brought closer to the sensor. It was observed that the output voltage from the sensor increases as the concentration of the smoke increases depending on the distance between the sensor and the smoke. Table 2 and Figure 10 show the response of the MQ-2 smoke sensor to the smoke from fire.

Table 2: Readings from the output of the smoke sensor

\begin{tabular}{|lrr|l|l|}
\hline \multicolumn{2}{|l|}{ Volt a g e } & $(\mathrm{v})$ & Distance $(\mathrm{cm})$ \\
\hline 2 &. & 8 & 2 \\
\hline 1 &. & 8 & 4 \\
\hline 1 &. & 3 & 6 & \\
\hline 1 &. & 0 & 1 & 0 \\
\hline 0 &. & 2 & 1 & 2 \\
\hline
\end{tabular}

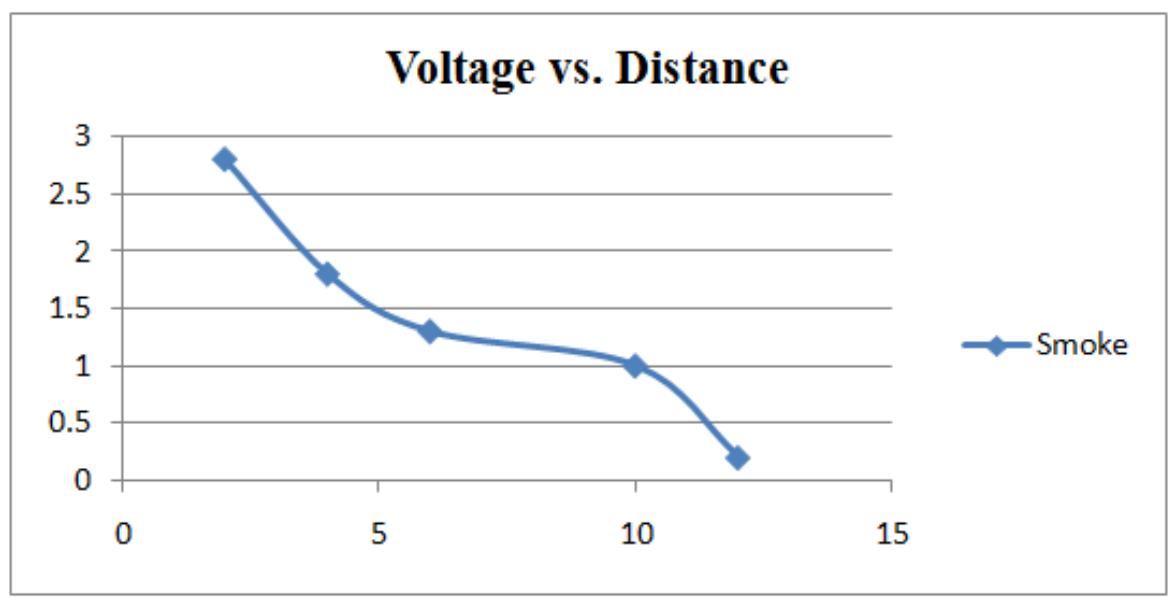

Fig 10: Graphical representation of the response of the smoke sensor to smoke from fire

\section{Performance Evaluation}

The table below shows the summary of the entire system performance as well as the tests carried out on the entire system to ascertain if it's working according to the desired objectives and specifications intended for it. The observations and results of the system are then recorded at room temperature, on introduction of fire close to the temperature sensor (LM35), and on introduction of smoke close to the smoke sensor (MQ-2).

The entire system is evaluated based on the tests, observations and results captured in the table below.

Table 3: System performance evaluation.

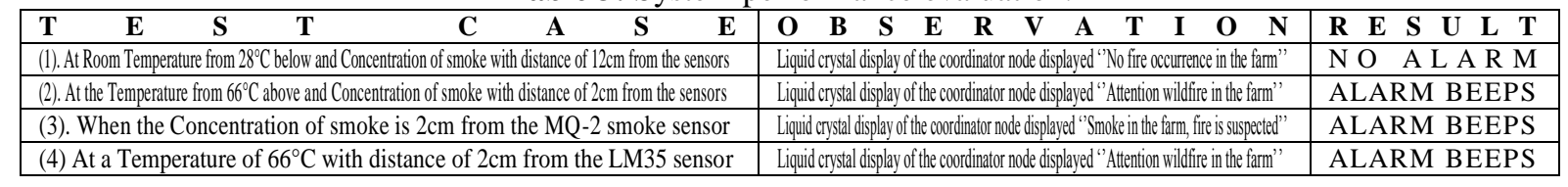

\section{Conclusion}

Modern communication technologies are employed to deal with emergencies. Monitoring and alert systems have incorporated the telecommunications infrastructure as an integral and basic part of its system. This project has introduced a viable alert and monitoring technique that promotes real time sensing and detection, monitoring, analysis of critical system information. These Monitoring and alert systems involves the application of engineering principles and techniques to the construction and design of efficient and high fidelity monitors which guarantees Increased timing accuracy thereby providing overall improvements in system performance (quality and efficiency). Wireless and mobile technologies are key components that would help enable users all over the world effectively manage and respond appropriately to fire hazards. This wireless communications would not only provide us with safe and accurate monitoring but also guarantees location independency.

The availability of GSM has enabled cost savings in monitoring and alert systems that depends on real time and has led to significant advances in capability and quality delivery. Monitoring systems saves lives by preventing industrial and home accidents, aiding search and rescue efforts, and speeding the delivery of 
emergency services and disaster relief. It also advances scientific aims such as weather forecasting, earthquake monitoring, and environmental protection and conservation.

This work proposes and focuses on the implementation of a low cost device that is very efficient, flexible, location independent while ensuring continuous, real time wildfire monitoring and alert thereby completely eliminatelags and errors irrespective of the volume and rate smoke sensed or detected. The system determines the smoke rate and then sends short message service (SMS) alert to the mobile phone of environmental personnel or relevant offices involved with wildfire control, thus enabling officers and personnel to effectively monitor and respond appropriately to emergency situations. This system is cost effective and user friendly and thus its usage is not restricted or limited to any class of users. It is a very efficient system and very easy to handle and thus provides great flexibility and serves as a great improvement over other conventional monitoring and alert systems.

\section{References}

[1]. Yanjun Li, Zhiwang, Yeqiong Song “' Wireless Sensor Network Design For Wildfire Monitoring”,National Laboratory of Industrial Control Technology Zhejiang University Hangzhou, China, Campus science Tigique-B.P239

[2]. Mohamed Hefeeda, MajidBagheri 'Forest Fire Modelling and Early Detection Using Wireless Sensor Network' School of Computing Science, Simon Fraser University, Canada 2009.volume 7,pp.169-224

[3]. P.S. Jadhaav, V.U Deshmukh " Forest Fire Monitoring System Based on ZIG-Bee Wireless Sensor Network", Volume 2, Number12, 2012, pp.1-6

[4]. Tuofiqul Islam, Syed Asif Abdullah, GolamSarowar "Enhanced Wireless control System for Smoke and Fire Detection"' International Journal of Computer and Electrical Engineering, Volume 5, Number 2, April 2013.PP.1-4

[5]. Yanjun LI, Zhi Wang, Yeqiong Song 'Wireless Sensor Network Design For Wildfire Monitoring', National Laboratory of Individual Control Technology Zhejiang University Hangzhou, China, Campus Scientifique-BP.239

[6]. UjwalParmar, Sharanjeet Singh " Comparative study of Zig-Bee, Bluetooth, and Wi-Fi Technology for constructing wireless Fire Alarm System', Volume 4, Number 4, September 2014,pp.893-897 